\title{
Sympathetic signaling facilitates progression of neuroendocrine prostate cancer
}

\author{
Shubham Dwivedi ${ }^{1,2}$, Maricris Bautista ${ }^{1,2}$, Sanskriti Shrestha ${ }^{1,2}$, Hussain Elhasasna ${ }^{3}$, Tanaya Chaphekar ${ }^{4}$, Frederick S. Vizeacoumar ${ }^{3}$ and $^{2}$ \\ Anand Krishnan (iD ${ }^{1,2}$
}

(c) Crown 2021

The progression of prostate cancer (PC) into neuroendocrine prostate cancer (NEPC) is a major challenge in treating PC. In NEPC, the PC cells undergo neuroendocrine differentiation (NED); however, the exact molecular mechanism that triggers NED is unknown. Peripheral nerves are recently shown to promote PC. However, their contribution to NEPC was not studied well. In this study, we explored whether sympathetic neurosignaling contributes to NED. We found that human prostate tumors from patients that later developed metastases and castration-resistant prostate cancer (CRPC), a stage preceding to NEPC, have high sympathetic innervations. Our work revealed that high concentrations of the sympathetic neurotransmitter norepinephrine (NE) induces NED-like changes in PC cells in vitro, evident by their characteristic cellular and molecular changes. The NE-mediated NED was effectively inhibited by the $A d r \beta 2$ blocker propranolol. Strikingly, propranolol along with castration also significantly inhibited the development and progression of NEPC in vivo in an orthotopic NEPC model. Altogether, our results indicate that the NE-Adr 32 axis is a potential therapeutic intervention point for NEPC.

Cell Death Discovery (2021)7:364 ; https://doi.org/10.1038/s41420-021-00752-1

\section{INTRODUCTION}

Prostate cancer (PC) is the second leading cancer diagnosed and the fifth leading cause of cancer-related deaths in men worldwide [1]. The mortality associated with PC often results from the development of treatment-resistant neuroendocrine PC (NEPC) [2]. In NEPC, prostate adenocarcinoma cells transdifferentiate into neuroendocrine cells $[3,4]$. However, the exact mechanism of neuroendocrine differentiation (NED) is unknown and, hence, effective therapies for NEPC are currently lacking. The average survival period of NEPC patients is around 7-15 months [2].

Recent studies demonstrated that peripheral nerves promote PC [5-7]. For example, denser autonomic innervation is associated with poor prognosis of PC $[5,6]$. In addition, several studies showed that the sympathetic neurotransmitter norepinephrine (NE) promotes PC migration and metastasis by activating adrenergic $\beta$-receptors $(\operatorname{Adr} \beta s)[8,9]$. Interestingly, a recent study demonstrated that Adr $\beta 2$ indeed promotes NED [10]. However, whether NE directly contributes to NED is not known. Understanding whether NE has any direct contribution to NED would expand potential therapeutic intervention points for NEPC spanning around NE biosynthesis, metabolism and NE-Adr $\beta$ axis. In this study, we explored whether NE has any direct potential to induce NED. Strikingly, we found that NE at supraphysiological concentrations induces the essential morphological and molecular features required for NED of PC cells. We revealed that the NEmediated NED involves $\operatorname{Adr} \beta 2$ signaling. Overall, our study indicates that targeting the NE-Adr 32 axis may prevent NEPC development and progression.

\section{RESULTS \\ Human prostate tumors are densely innervated by sympathetic nerves}

We examined the sympathetic nerve distribution in eight human prostate adenocarcinoma and corresponding normal adjacent tissues to understand whether sympathetic axonogenesis occurs in prostate tumors. Tyrosine hydroxylase (TH) was used for staining the sympathetic nerves. Co-staining experiments with $\mathrm{TH}$ and the pan-neuronal marker $\beta \mathrm{III}$ tubulin confirmed that $\mathrm{TH}$ specifically stains nerve fibers (Supplementary Fig. S1). We found that four out of the eight adenocarcinoma samples analyzed had higher sympathetic innervations (Fig. $1 \mathrm{~A}$ and Table 1). The associated clinical information data from ACRB revealed that three out of those four patients that demonstrated higher sympathetic innervations in tumors had developed metastasis later. Most importantly, two of them developed castrationresistant prostate cancer (CRPC) (Table 1). Although this is a low sample size, it indicates the propensity of sympathetic signaling in facilitating PC progression. Increased tumor innervations also indicate that active axonogenesis occurs in PC. We found that the newly formed axons make specific contacts with cancer cells, indicating their direct interaction with each other (Fig. 1B). We then examined the expression of $\operatorname{Adr} \beta 1, \operatorname{Adr} \beta 2$, and $\operatorname{Adr} \beta 3$

\footnotetext{
${ }^{1}$ Department of Anatomy, Physiology, and Pharmacology, College of Medicine, University of Saskatchewan, Saskatoon, SK S7N 5E5, Canada. ${ }^{2}$ Cameco MS Neuroscience Research Centre (CMSNRC), Saskatoon, SK S7K 0M7, Canada. ${ }^{3}$ Division of Oncology, College of Medicine, University of Saskatchewan, Saskatoon, SK S7N 5E5, Canada. ${ }^{4}$ Department of Arts and Science, University of Tennessee, Knoxville, TN, USA. ${ }^{凶}$ email: anand.krishnan@usask.ca
}

Received: 19 August 2021 Revised: 27 September 2021 Accepted: 1 November 2021

Published online: 22 November 2021 

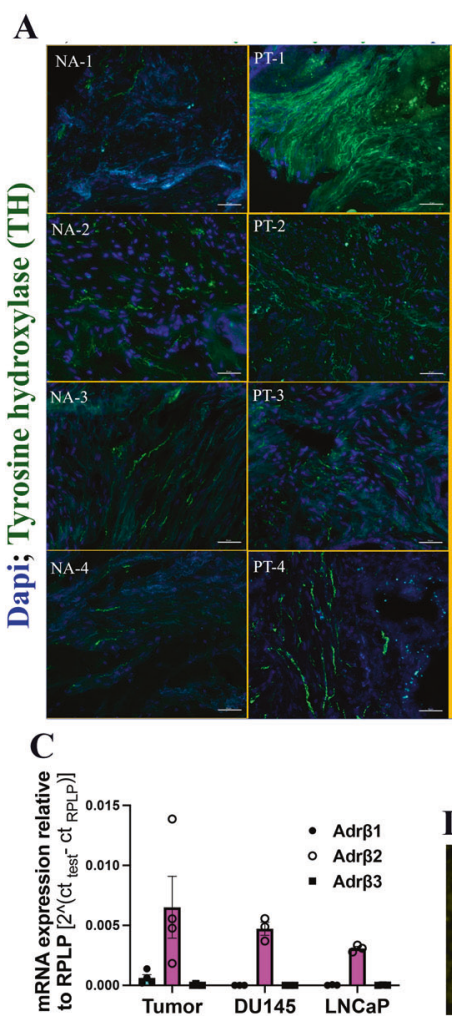

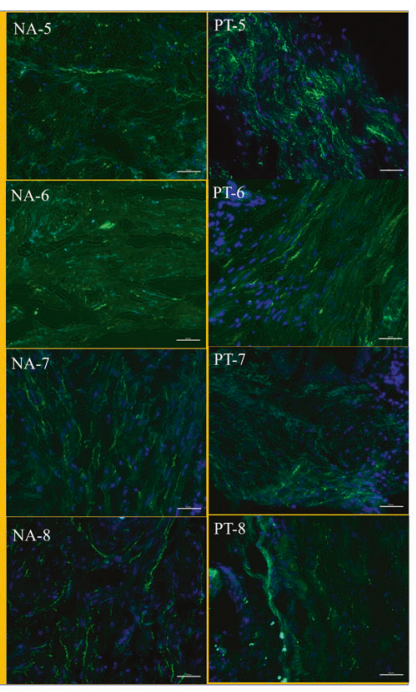

D

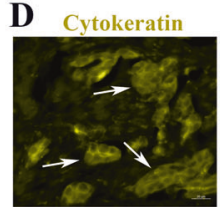

B
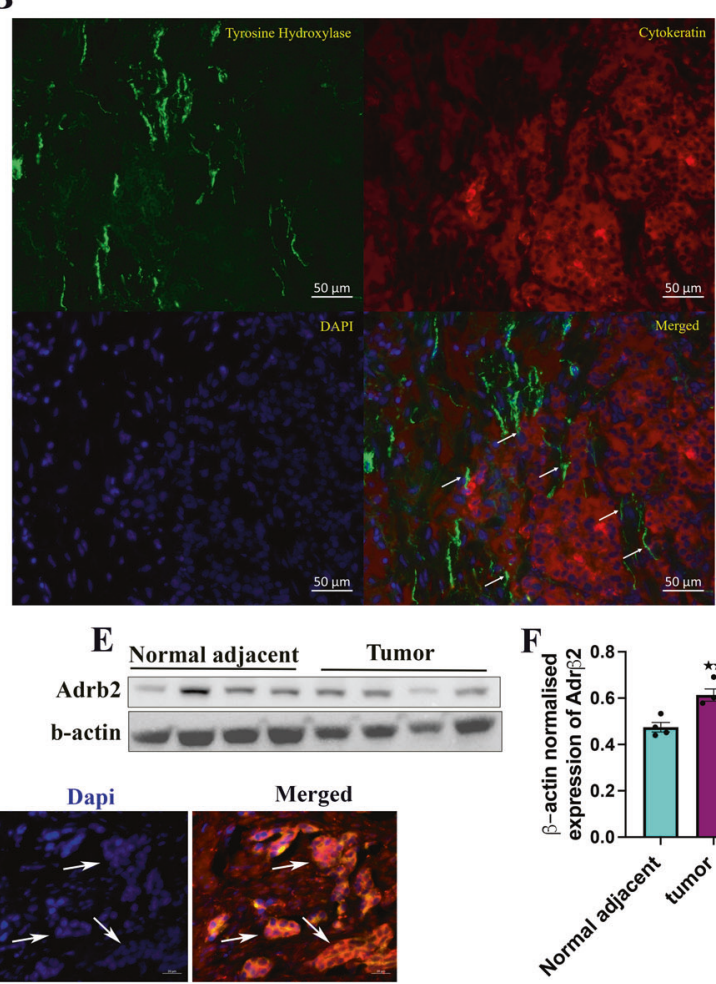

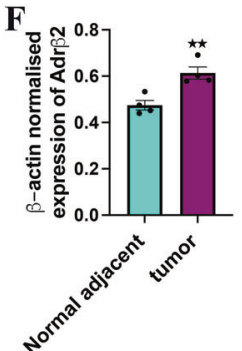

Fig. 1 Distribution of sympathetic nerves and adrenergic receptors in human prostate tumors and corresponding normal adjacent tissues. A Tyrosine hydroxylase (TH: green) staining showing the distribution of sympathetic nerves in eight prostate tumors (PT) and corresponding normal adjacent tissues (NA). Dapi (blue) was used for staining the nuclei. Scale bar, $50 \mu \mathrm{m}$. B Co-staining of TH (green) and pan-cytokeratin (red; cancer cells) shows the physical contacts between sympathetic axons and cancer cells (shown using white arrows in the merged image). Scale bar, $50 \mu \mathrm{m}$. C qRT-PCR analysis of Adr $\beta$ receptor mRNAs in human prostate tumors and the PC cell lines, DU145 and LNCaP, shows relatively high expression of $\operatorname{Adr} \beta 2$ ( $n=4$ for tumors, $n=3$ for DU145 and LNCaP cells). D Immunostaining showed the expression of Adr $\beta 2$ (red) in cancer cells (yellow: cytokeratin) in human prostate tumor. The corresponding areas are shown using arrows. Scale bar, $20 \mu \mathrm{m}$. E Western blotting shows the expression of $\mathrm{Adr} \beta 2$ in human prostate tumors and corresponding normal adjacent tissues. $\beta$-Actin was used as the loading control. F Quantification of " $E$ " shows upregulation of Adr $\beta 2$ in human prostate tumors. The data are presented as mean \pm SEM $(n=4)$ and statistically analyzed using standard Student's $t$-test (unpaired, two-tailed). $p<0.05$ was considered significant, where ${ }^{* *} p<0.01$ compared to the normal adjacent values.

Table 1. Clinical information associated with the prostate tumors and adjacent normal tissues used for the study.

\begin{tabular}{|c|c|c|c|c|c|c|}
\hline Sample number & Diagnosis & Gleason score & $\begin{array}{l}\text { Metastatic } \\
\text { occurrence }\end{array}$ & $\begin{array}{l}\text { Development of } \\
\text { CRPC within } 10 \\
\text { years of surgery }\end{array}$ & $\begin{array}{l}\text { Sympathetic } \\
\text { innervations in } \\
\text { adjacent normal } \\
\text { tissues }\end{array}$ & $\begin{array}{l}\text { Sympathetic } \\
\text { innervations in } \\
\text { tumor tissues }\end{array}$ \\
\hline 2 & Adenocarcinoma & 7 & $\begin{array}{l}\text { Lung Lymph Node, } \\
\text { Distant Pelvic Soft } \\
\text { Tissue, Bone }\end{array}$ & $\sqrt{ }$ & 3146.097 & 5385.674 \\
\hline 5 & Adenocarcinoma & 7 & - & & 5235.397 & 7027.38 \\
\hline 6 & Adenocarcinoma & 8 & - & & 3788.026 & 2950.441 \\
\hline 7 & Adenocarcinoma & 7 & - & & 6975.41 & 4096.858 \\
\hline 8 & Adenocarcinoma & 7 & - & & 2462.88 & 1823.715 \\
\hline
\end{tabular}

receptors in prostate tumors and PC cell lines and found that $A d r \beta 2$ is relatively highly expressed in them, suggesting that Adr $\beta 2$ may be the receptor mediating the sympathetic nerve-tumor interaction (Fig. 1C, D). Quantification of Adr $\beta 2$ expression in tumor samples and corresponding normal adjacent tissues revealed that $A d r \beta 2$ is overexpressed in tumors (Fig. 1E, F). Overall, our results suggest that newly formed sympathetic fibers may play critical roles in PC progression, in particular its transition to advanced stages such as CRPC, by establishing a direct interaction with cancer cells. 


\section{NE induces NED-like morphological changes in PC cells}

Based on our observation that prostate tumors acquire higher sympathetic innervations, we asked whether the sympathetic neurotransmitter NE has any direct role in promoting PC, especially its advancement into NEPC. NED is a pre-requisite for NEPC. Therefore, we focus our studies on NE's potential contribution to NED. We used both androgen receptor negative $\left(\mathrm{AR}^{-}\right) \mathrm{DU} 145$ and $\mathrm{AR}^{+} \mathrm{LNCaP}$ cells, representing two spectra of PC cells, for our experiments. A previous pre-clinical study reported that early-stage prostate tumors achieve high concentrations (supraphysiological) of NE from local nerves [6]. Therefore, we considered physiologically relevant $(10 \mu \mathrm{M})$ and supraphysiological ( $50 \mu \mathrm{M}$ and above) concentrations of NE for our initial experiments. Strikingly, we observed that NE at all supraphysiological concentrations $(50-300 \mu \mathrm{M})$ induced NED-like morphological changes in PC cells, evident from the development of neurite-like extensions and compact cell bodies (Fig. 2A, B). Although neurite-like extensions were evident in DU145 cells at $50 \mu \mathrm{M}$ NE treatment, compact cell bodies were much apparent only from $100 \mu \mathrm{M}$ NE onwards. At the same time, both increased neurite-like extensions and compact cell bodies appeared in LNCaP cells in response to $50 \mu \mathrm{M}$ NE onwards. We did not find such morphological changes in PC cells at a lower concentration of $\mathrm{NE}$, such as $10 \mu \mathrm{M}$, even after $96 \mathrm{~h}$ treatment (Supplementary Fig. S2). We also noted that, at a dose of $300 \mu \mathrm{M}$ NE and above, the cells lose viability and detach from the surface (see below for additional details). Overall, our result indicates that NE induces NED-like morphological changes in PC cells, regardless of their AR status.

\section{NE-induced NED-like changes does not alter the viability of PC cells}

We next examined the viability of NE-treated cells to determine at what supraphysiological concentrations it induces NED-like morphological changes, while retaining the cell viability intact. For this, we performed an 3-(4,5-Dimethylthiazol-2-yl)-2,5-Diphenyltetrazolium Bromide (MTT)-based cell viability assay in DU145 cells after treating them with NE in a dose- and time-dependent manner. Our assay revealed a $\mathrm{CC}_{50}$ value of $494,272,207$, and $214 \mu \mathrm{M}$ at 24, 48, 72, and $96 \mathrm{~h}$ NE treatment, respectively (Supplementary Fig. S3A). Combined with our previous result, it indicates that a dose range of $50-200 \mu \mathrm{M}$ NE induces NED-like morphological changes, and at the same time, retains more than $50 \%$ cell viability for a period of $24-96 \mathrm{~h}$. Our examination of propidium iodide (PI) live/dead cell staining, where the dead cells only would take up the dye, also did not show difference in $\mathrm{PI}$ staining between the control and up to $200 \mu \mathrm{M}$ NE treatment over a period of $48 \mathrm{~h}$, further confirming that NE at supraphysiological concentrations up to $200 \mu \mathrm{M}$ is not cytotoxic to PC cells (Supplementary Fig. S3B). Although this result demonstrated that a single pulse of up to $200 \mu \mathrm{M}$ NE induces NED in PC cells without affecting their viability, prostate tumors are likely to receive continuous pulses of NE from denser sympathetic innervations. Therefore, we tested the effect of frequent pulses of NE on NED by replenishing the culture media with fresh NE (50-200 $\mu \mathrm{M})$ every $24 \mathrm{~h}$ for 7 days. We found NED-like morphology, such as neuritelike extensions and compact cell bodies, in these cultures at all the time points (96 and $168 \mathrm{~h}$ ) and concentrations (50, 100, $200 \mu \mathrm{M})$ tested (Supplementary Fig. S4). Importantly, our PI live/dead cell
A
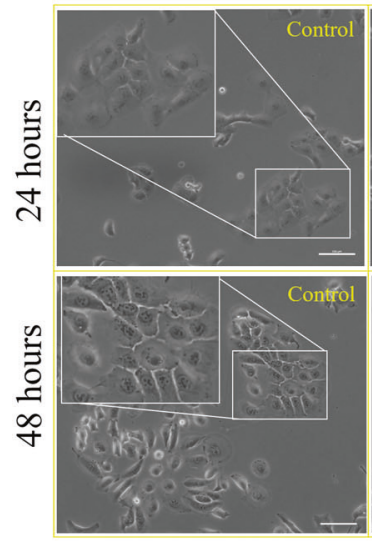

B
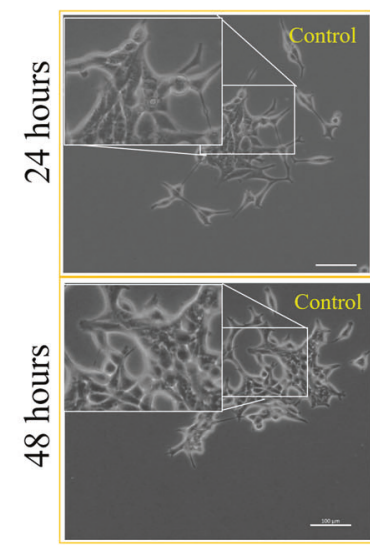

DU145 cells
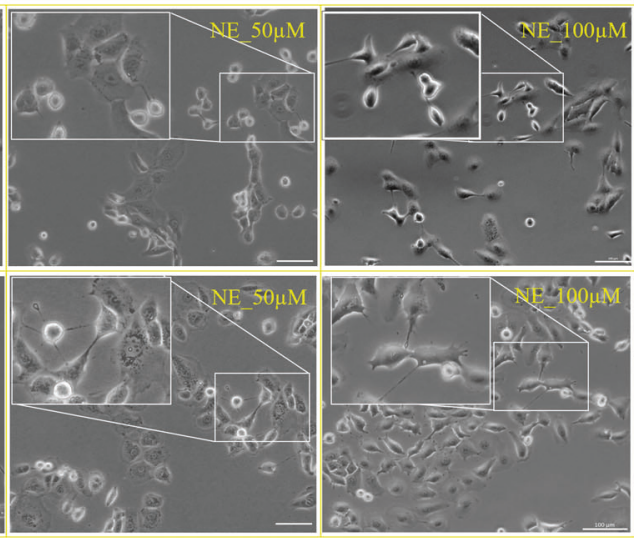

LNCaP cells
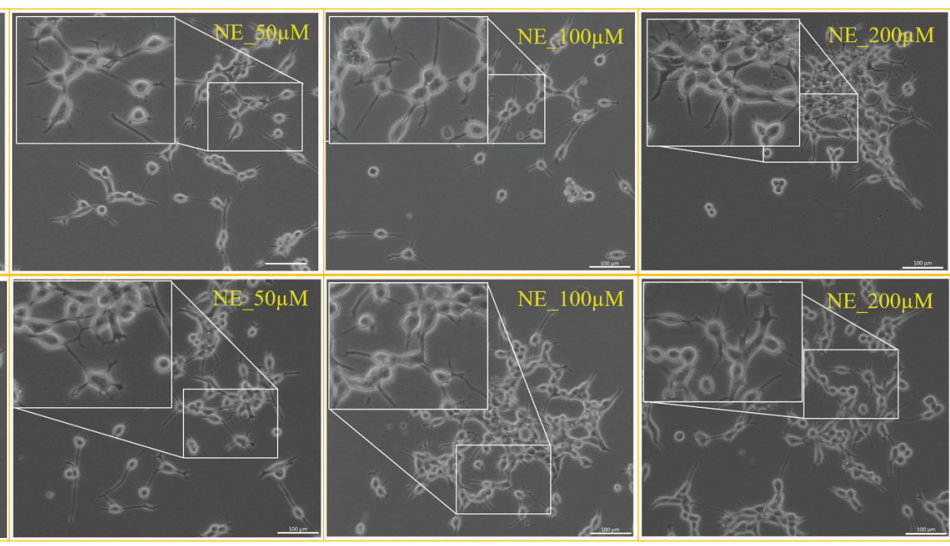
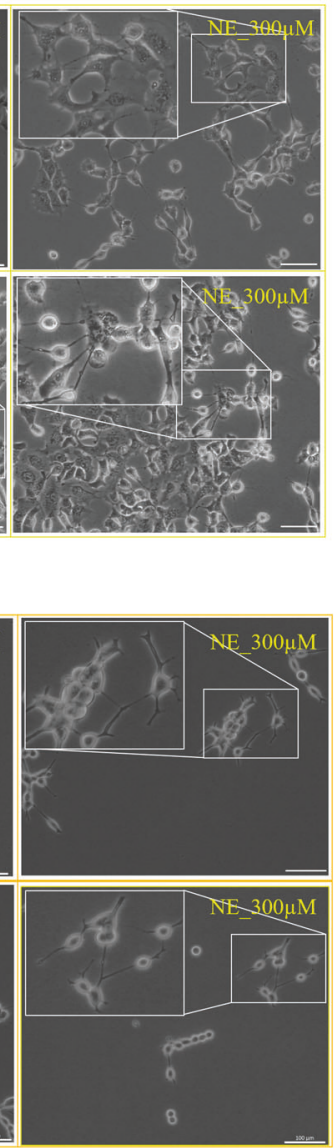

Fig. 2 NE induces NED-like morphological changes in PC cells. A, B Dose- and time-dependent NE treatment induces NED-characteristic features, such as neurite-like extensions and compact cell bodies, in DU145 cells (A) and LNCaP cells (B). Magnified representations are provided in the insets. Scale bar, $100 \mu \mathrm{m}$. 
A mRNA expression in DU145 cells: $24 \mathrm{~h}$
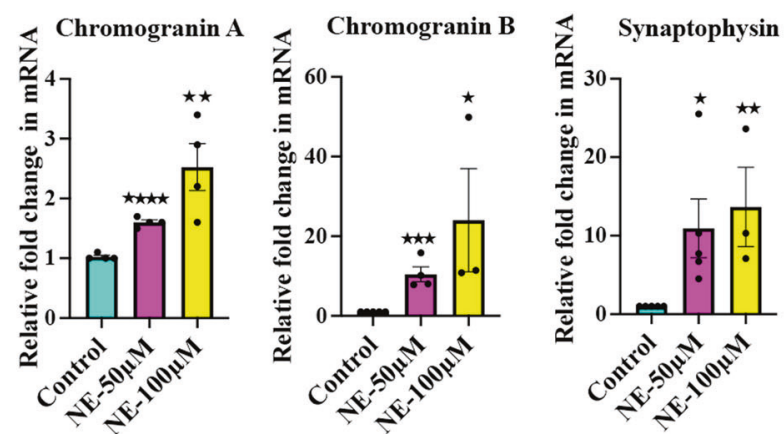

\section{B mRNA expression in DU145 cells: $48 \mathrm{~h}$}
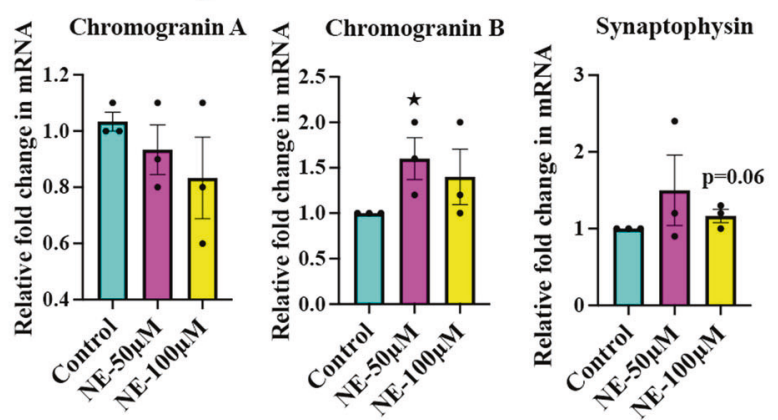

\section{C mRNA expression in LNCaP cells: $24 \mathrm{~h}$}
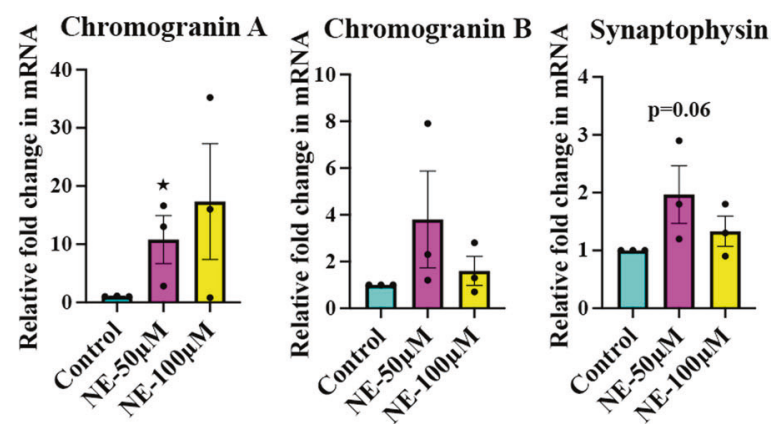

\section{D mRNA expression in LNCaP cells: $48 \mathrm{~h}$}
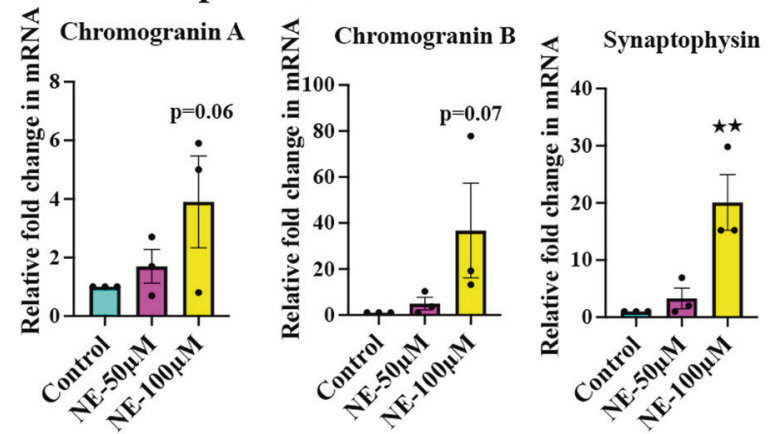

Fig. 3 NE induces NED markers in PC cells. A, B Quantitative real-time PCR shows that NE induces the upregulation of the NED markers CHGA, CHGB, and SYP in DU145 cells at $24 \mathrm{~h}(\mathbf{A})$ and $48 \mathrm{~h}(\mathbf{B})$. The data are presented as mean \pm SEM $(n=3$ minimum) and statistically analyzed using standard Student's $t$-test (unpaired, one-tailed). $p<0.05$ was considered significant, where ${ }^{*} p<0.05,{ }^{* *} p<0.01,{ }^{* * *} p<0.001$, and ${ }^{* * * *} p<0.0001$ compared to control. C, D Quantitative real-time PCR shows that NE induces the upregulation of the NED markers CHGA, CHGB, and SYP in LNCaP cells at $24 \mathrm{~h}(\mathrm{C})$ and $48 \mathrm{~h}(\mathrm{D})$. Data are presented as mean \pm SEM $(n=3)$ and statistically analyzed using standard Student's $t$-test (unpaired, one-tailed). $p<0.05$ was considered significant, where ${ }^{*} p<0.05$ and ${ }^{* *} p<0.01$ compared to control.

staining did not show cytotoxicity in these cultures, indicating that even continuous pulses of supraphysiological NE is not cytotoxic, but can trigger, and perhaps maintain, transdifferentiation of PC cells.

\section{NE induces characteristic molecular changes involved with NED in PC cells}

We next asked whether the NED-like morphology induced by NE is true NED by examining the NED-characteristic molecular changes in PC cells. For this, we examined the mRNA expression of the well-known NED markers chromogranin A (CHGA), chromogranin B (CHGB), and synaptophysin (SYP) in DU145 and LNCaP cells after NE treatment. We found a dose-dependent upregulation of CHGA, CHGB, and SYP mRNAs in DU145 cells at $24 \mathrm{~h}$, whereas there was a slight fluctuation in their levels at $48 \mathrm{~h}$ (Fig. 3A-B). Strikingly, NE at the lower spectrum of the supraphysiological concentrations tested, such as $50 \mu \mathrm{M}$, consistently induced the upregulation of CHGB and SYP at both treatment time points. Similarly, both $50 \mu \mathrm{M}$ and $100 \mu \mathrm{M} \mathrm{NE}$ induced the upregulation of all three markers in LNCaP cells at $24 \mathrm{~h}$ and $48 \mathrm{~h}$, confirming that NE induces NED-characteristic molecular changes in PC cells (Fig. 3C-D).

We then examined the protein expression of $\mathrm{CHGB}$ and SYP by immunostaining in PC cells after NE treatment. We observed the characteristic granular staining of both CHGB and SYP in DU145 cells after $50 \mu \mathrm{M}$ NE. However, they were absent in control cells, indicating that NE specifically upregulates the NED markers expression (Fig. 4A). The LNCaP cells showed mild basal expression of these markers, evident from their uniform cytoplasmic staining in control cells. However, $50 \mu \mathrm{M} \mathrm{NE}$ accentuated their staining intensity in the cells confirming that $\mathrm{NE}$ induces the expression of these NED markers (Fig. 4B). Overall, our result indicates that $\mathrm{NE}$, even at a lower spectrum of the supraphysiological concentration, initiates true NED in PC cells.

\section{Adr $\beta$ s are involved with NE-mediated NED}

We next examined whether $\operatorname{Adr} \beta 2$ play a role in NE-mediated NED. While $50 \mu \mathrm{M}$ NE induced the upregulation of CHGB and SYP mRNAs in both DU145 and LNCaP cells indicating initiation of transdifferentiation, pre-treatment with propranolol (an Adr $\beta 1$ and $A d r \beta 2$ antagonist) inhibited their upregulation (Fig. S5A, B). Similarly, immunostaining showed that propranolol prevents NE-mediated induction of CHGB and SYP remarkably in DU145, whereas its effect was moderate in LNCaP cells (Fig. S5C, D). As our earlier results showed that $A d r \beta 2$ has a comparatively higher expression in these cells, the propranolol-mediated inhibition indicates that NE induces NED through activation of Adr $\beta 2$ receptors.

\section{Propranolol inhibits the development and progression of NEPC}

We next examined the effect of Adr $\beta 2$ inhibition in NEPC development. We tested this by inhibiting Adr $\beta 2$ using propranolol in an orthotopic NEPC model. We used the well-established NEPC cell line, LASCPC-01, to generate the NEPC orthotopic model. Control animals were treated with saline, whereas two other groups were treated with either castration or castration + propranolol. We performed castration to mimic androgen deprivation therapy (ADT), which is a standard treatment approach in the PC clinics prior to advanced stages, such as 

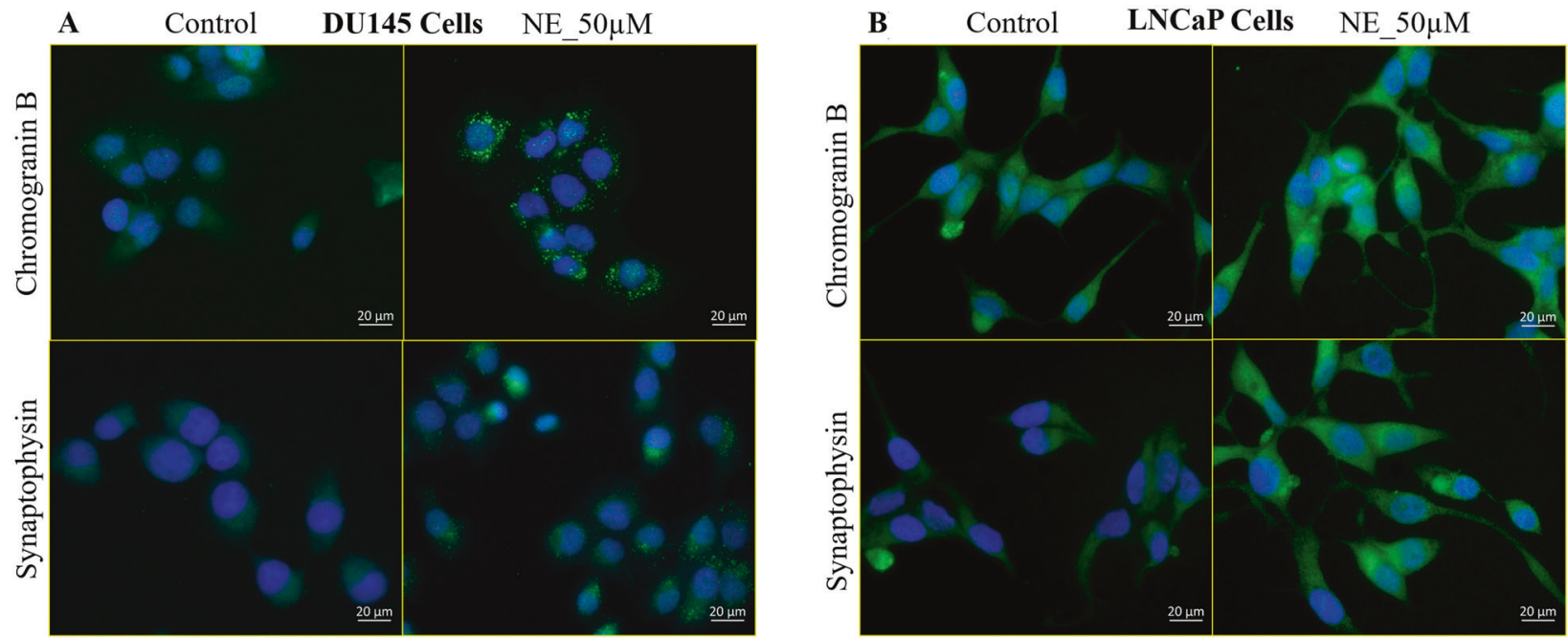

Fig. 4 NE induces NED markers in PC cells. A Immunostaining shows that $50 \mu M$ NE induces the expression of the NED markers CHGB and SYP in DU145 cells at $24 \mathrm{~h}$. Both CHGB and SYP show characteristic granular staining after NE treatment. B $50 \mu \mathrm{M}$ NE induces the expression of CHGB and SYP in LNCaP cells at $24 \mathrm{~h}$. The control cells display weak basal expression of CHGB and SYP, but NE treatment upregulates the expression of these markers, evident by their increased staining intensity. Scale bar, $20 \mu \mathrm{m}$.

NEPC. Propranolol treatment lasted for 21 days. Interestingly, we found that all animals in the saline control and castration-alone group developed tumors; however, only one out of the four animals in the castration + propranolol group developed tumor (Fig. 5A, B). Our results thus showed that, while castration alone had a significant effect in lowering the tumor size, its combination with propranolol provides enhanced protection in terms of NEPC development and progression, indicating that $\mathrm{Adr} \beta 2$ inhibition is an ideal therapeutic strategy for NEPC.

\section{DISCUSSION}

Nerve dependence of PC has gained much attention recently [7, 11, 12]. For example, studies showed that depletion of autonomic nerves suppress PC. Follow-up studies revealed a critical involvement of stromal cells in nerve-dependent PC growth [5]. For instance, selective knockdown of $\operatorname{Adr} \beta 2$ or $\operatorname{Adr} \beta 3$ in stromal cells was shown to suppress PC in animal models $[5,6]$. Sympathetic nerve-mediated Adr 32 activation in endothelial cells and resulting enhanced angiogenesis also indirectly promotes nerve-dependent PC growth [6]. Nerves directly alter PC cell dynamics by inducing perineural invasion where PC cells use nerves as physical cues to invade and metastasize [13]. Nerves are also equipped with tumor regulatory machineries, such as active tumor suppressor and DNA repair networks, in modulating tumor dynamics [14-16]. However, whether nerves play a direct role in promoting NEPC has not been studied. In this study, we report the potential role of $\mathrm{NE}-\mathrm{Adr} \beta 2$ axis in inducing $\mathrm{NED}$ and NEPC.

A previous study demonstrated that a potent $\operatorname{Adr} \beta 2$ agonist isoproterenol induces proliferation of PC cells [17]. Therefore, we initially examined whether NE induces PC cell proliferation, but our experiments failed to demonstrate significant cell proliferation in response to $10-30 \mu \mathrm{M} \mathrm{NE}$, a standard dose used in the literature (data not shown). This led to our attention to a pre-clinical study, which showed that high-grade prostatic intraepithelial neoplasia (HGPIN), an early-stage PC, acquires higher levels of NE compared to healthy prostate [6]. For example, it was shown that mouse HGPIN expresses $\sim 120 \mathrm{ng} / \mathrm{mg}$ protein of NE [6]. Inclined to this finding, we and others observed that human prostate tumors possess denser sympathetic innervations [5]. We then estimated that a $40 \mathrm{mg}$ human prostate tumor expresses a total of $4 \mathrm{mg}$ protein. Considering that a complete human prostate tumor weighs a minimum of $1-2 \mathrm{~g}$, the total protein concentration that could be achieved in the tumor is around 100-200 mg, which can consist of $12-24 \mu \mathrm{g}$ of NE. Then, $100 \mu \mathrm{M}$ of NE in $1 \mathrm{ml}$ culture offers $16 \mu \mathrm{g} \mathrm{NE}$, which closely matches the tumor-level, supraphysiological, NE. Interestingly, our experiments using the supraphysiological levels of NEinduced NED in both $\mathrm{AR}^{+}$and $\mathrm{AR}^{-} \mathrm{PC}$ cells. As NED often leads to NEPC, our finding indicates that aberrant sympathetic activity, and the resulting increased $\mathrm{NE}$ signaling, might contribute to NEPC.

Androgen (testosterone) is a major growth factor for PC and ADT is a standard and effective treatment for PC [18]. However, PC eventually develops resistance to ADT, undergoes NED, and emerges as treatment-resistant NEPC. Our findings that supraphysiological NE could induce NED of PC cells suggest that NE might serve as an alternate growth factor for $P C$, when androgen is deprived, resulting in the transition of PC into NEPC. DU145 cells we used in our experiments lack AR and LNCaP cells, although they express AR, were cultured in androgen-lacking media. Thus, our culture conditions simulated an ADT environment and the effect of NE at these culture conditions in inducing NED supports our argument. We did not, however, examine the effect of NE in inducing NED in androgen enriched conditions.

We found that NE-mediated NED is dependent on Adr 32 . A recent study using a subcutaneous PC xenograft model also demonstrated that $\operatorname{Adr} \beta 2$ is involved with NED [10]. In general, cutaneous structures have less autonomic innervations compared to internal organs and, hence, the triggering force of $\mathrm{Adr} \beta 2$ activation in subcutaneous PC models is not clear and less likely to be NE. Therefore, NE being the major catecholamine available in the prostate, our finding of its potential to induce NED warrants special attention. Our experiments showed that propranolol inhibits NEdriven NED. Although propranolol targets both $\operatorname{Adr} \beta 1$ and $\operatorname{Adr} \beta 2$, our mRNA analysis showed that Adr $\beta 2$ is highly expressed, compared to Adr $\beta 1$, in PC cells and prostate tumors, and hence we believe that $A d r \beta 2$ might be critical for NE-driven NED.

Overall, our study, for the first time, showed that supraphysiological concentrations of NE facilitates NED of PC cells through activation of $A d r \beta 2$. The prostate is supplied by hypogastric and pelvic nerves, providing adrenergic and cholinergic innervations, respectively. The actions of the cholinergic neurotransmitter acetylcholine (Ach) and nerve-derived neurotrophic factors, such as nerve growth factor and brain-derived neurotrophic factor in the prostate milieu might influence the action of NE on PC cells 

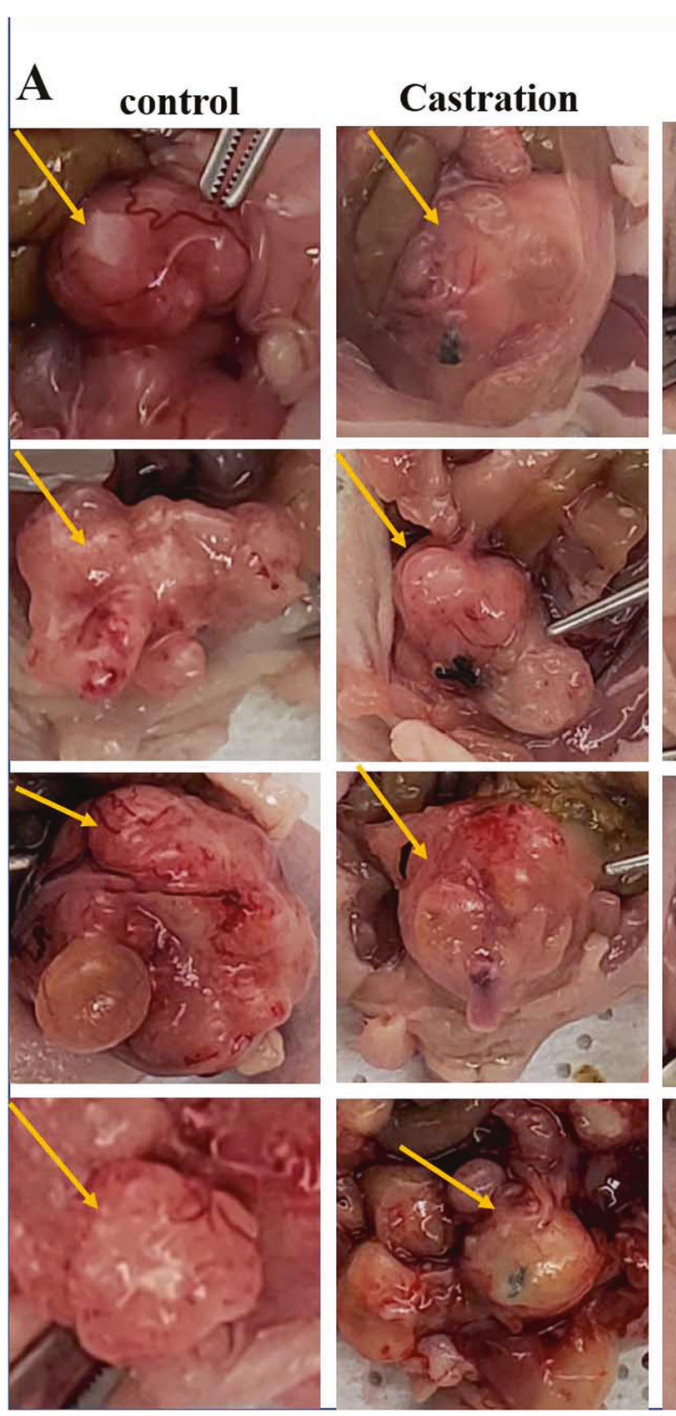

Castration
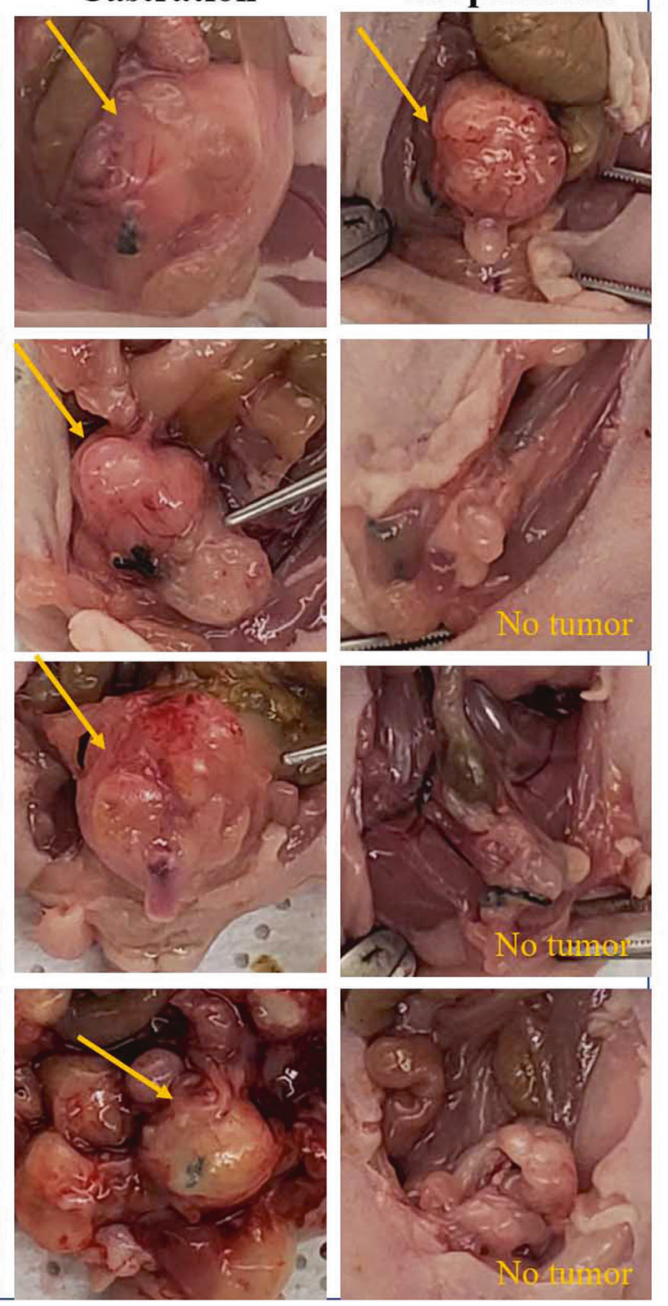

B

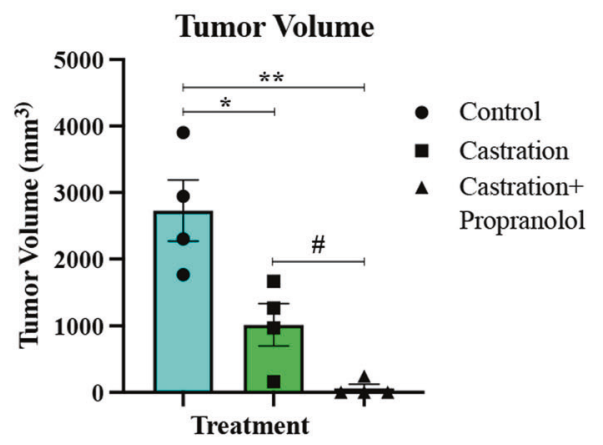

Fig. 5 Propranolol inhibits the development and progression of NEPC in orthoptic NEPC models. A Individual tumors from the saline control, castration-alone, and castration + propranolol groups show remarkable inhibition of NEPC in the castration + propranolol group. B Quantification of "A" shows significant reduction in tumor growth in castration + propranolol group compared to the saline control and castration-alone group. Data are presented as mean \pm SEM ( $n=4$ animals/group) and statistically analyzed using Standard " $t$ " test (unpaired, two-tailed). $P<0.05$ was considered significant, where ${ }^{*} p<0.05,{ }^{* *} p<0.01$ compared to control group and \#p<0.05 compared to castrationalone group.

in vivo. Further investigations on the fate of cancer cells in response to the co-ordinated actions of $\mathrm{NE}$, Ach, and neurotrophins may reveal an in-depth understanding of the nature of NEmediated NED in vivo. Having said that, our in vivo studies provide strong evidence that $A d r \beta 2$ inhibition along with castration has superior benefit in managing NEPC. Overall, our findings indicate that NE- Adr 32 axis is an ideal therapeutic intervention point for NEPC.

\section{MATERIALS AND METHODS \\ Cells, chemicals, and reagents}

The PC cell lines DU145 (ATCC HTB81) and LNCaP (ATCC CRL1740) were purchased from ATCC. NE bitartrate (A9512), propranolol hydrochloride (537075) and PI (P4170) were procured from Millipore Sigma. Trizol (15596018), cDNA synthesis kit, Dulbecco's modified Eagle medium (DMEM)/F12 media (11330032), and fetal bovine serum (12483020) were purchased from Life Technologies. Antibiotic/antimycotic solution (SV3007901) was purchased from HyClone. All other chemicals used were of analytical grade.

\section{Nerve innervation studies in human prostate tumors}

Human tissue studies were performed after receiving approval from the Biomedical Research Ethics Board at the University of Saskatchewan. Fresh, frozen human prostate adenocarcinoma tissues were procured from the Alberta Cancer Research Biobank (ACRB). They were fixed in Zamboni's fixative overnight at $4{ }^{\circ} \mathrm{C}$ and further incubated overnight in $20 \%$ sucrose solution. The tissues were then embedded in optimal cutting temperature (OCT) compound and allowed to freeze, followed by $12 \mu \mathrm{m}$ thick sections taken on slides. The generated sections were blocked for $30 \mathrm{~min}$ using $5 \%$ donkey serum containing $0.3 \%$ Triton $\mathrm{X}-100$. The sections were then colabeled with primary antibodies against TH (rabbit pAb; AB152, Sigma) and $\beta I I I$ tubulin (chicken pAb; AB9354, Sigma) for $1 \mathrm{~h}$, followed by incubation with a cocktail of anti-rabbit Alexa Fluor ${ }^{\circledR} 488$ Conjugate (A11034, ThermoFisher Scientific) and anti-chicken Alexa Fluor ${ }^{\circledR} 647$ (A21449, ThermoFisher Scientific) secondary antibodies for $1 \mathrm{~h}$ at room temperature. Sections were then mounted using slow-fade DAPI (S36973, Life Technologies) and images captured using Axio Observer 7 (inverted bright-field/fluorescence microscope, Carl Zeiss, Germany). Quantification of nerve fibers was done manually in a blinded manner by tracing the fibers using Fiji software.

To investigate nerve-cancer cell interface, some sections were also colabeled with primary antibodies against $\mathrm{TH}$ and cytokeratin (mouse mAb; 
MA1-82041, ThermoFisher Scientific, CA) for $1 \mathrm{~h}$, followed by incubation with a cocktail of anti-rabbit Alexa Fluor ${ }^{\circledR} 488$ Conjugate and anti-mouse Alexa Fluor 546 (A21045, ThermoFisher Scientific, CA) secondary antibodies for $1 \mathrm{~h}$ at room temperature.

\section{Morphological assessment of NED features}

The PC cells were cultured in DMEM/F12 media containing $10 \%$ fetal bovine serum and penicillin and streptomycin cocktail $(50 \mathrm{U} / \mathrm{ml})$ at $37^{\circ} \mathrm{C}$ and $5 \% \mathrm{CO}_{2}$ conditions. For the morphological assessment of NED occurrence, $5 \times 10^{4}$ cells/well were seeded in six-well plates and defined treatments were given after overnight incubation. The characteristic NED features, such as compact cell bodies and neurite-like extensions, were then evaluated at 24 and $48 \mathrm{~h}$ using Axio Observer 7.

\section{Cell viability}

Cell viability was assessed using both MTT assay (quantitative method) and PI staining (qualitative method). MTT assay was performed as done previously [19]. Briefly, $5 \times 10^{3}$ cells/well were seeded in a 96-well plate and incubated overnight. The cells were then given specific treatments and incubated for 24-96 $\mathrm{h}$. The media was then removed and $1 \mathrm{mg} / \mathrm{ml}$ of MTT solution $(100 \mu \mathrm{l})$ was added to each well followed by $4 \mathrm{~h}$ incubation at $37{ }^{\circ} \mathrm{C}$ and $5 \% \mathrm{CO}_{2}$ conditions. After the incubation, the MTT solution was removed and the formazan crystals formed were dissolved in $100 \mu \mathrm{l}$ of dimethyl sulfoxide. The absorbance of the solution was then read at 570 and $630 \mathrm{~nm}$ (for background correction) using SpectraMax M2 (Molecular Devices, San Jose, CA). The percentage change in cell viability was calculated compared to corresponding controls.

For PI staining, $5 \times 10^{4}$ cells/well were seeded in a six-well plate and incubated overnight at $37{ }^{\circ} \mathrm{C}$ and $5 \% \mathrm{CO}_{2}$ conditions. The cells were then treated with varying concentrations of NE for 8,24 , and $48 \mathrm{~h}$, followed by the media removed and $750 \mu \mathrm{l}$ of freshly prepared PI solution $(10 \mu \mathrm{g} / \mathrm{ml})$ added to the plate. The plates were then incubated at $37^{\circ} \mathrm{C}$ for $10 \mathrm{~min}$ and the images captured using Axio Observer 7.

\section{Real-time quantitative reverse-transcription PCR}

Total RNA was isolated using TRIzol reagent (15596018, Life Technologies) and quantified using ND-1000 spectrophotometer (NanoDrop Technologies, USA). Then, $500 \mathrm{ng}$ of total RNA was converted into CDNA using a CDNA synthesis kit (4368813, Applied Biosystems) as per the manufacturer's instructions. The CDNAs were amplified using the specific primers mentioned below, and by using PowerUp ${ }^{\mathrm{TM}}$ SYBR $^{\mathrm{TM}}$ Green Master Mix (A25741, Applied Biosystems). The CDNA amplifications were done in QuantStudio ${ }^{\mathrm{TM}} 3$ Real-Time PCR System (Applied Biosystems). All reactions were performed in triplicate, and the gene expressions were normalized to the house keeping gene, RPLP.

\begin{tabular}{ll}
\hline Primer & Sequence (5'-3') \\
Chromogranin A Forward & GGGATACCGAGGTATGAAATG \\
Chromogranin A Reverse & TCTCCTCGGAGTGTCTCAAA \\
Chromogranin B Forward & GGATGAGGAGGACAAGAGAAC \\
Chromogranin B Reverse & CCCTCTCTTCCTCACTITCTC \\
Synaptophysin Forward & CGTGTTGCCTCCTCTACT \\
Synaptophysin Reverse & GCATGGGCCCTTGTTATC \\
Adrß1 Forward & CAA TGT GCT GGT GAT CG \\
Adrß1 Reverse & CCA GGG ACA TGA TGA AGA \\
Adrß2 Forward & AGA CCT GCT GTG ACT TCT \\
Adrß2 Reverse & CTG AAA GAC CCT GGA GTA GA \\
Adrß3 Forward & GCT GGT TGC CCT TCT Tा \\
Adrß3 Reverse & GCA TAA CCT AGC CAG TTC AG \\
RPLP Forward & AGCCCAGAACACTGG TCT \\
RPLP Reverse & ACTCAG GATTCAATGGTCC \\
\hline
\end{tabular}

\section{Immunofluorescence}

NE-treated cells were fixed for $15 \mathrm{~min}$ using $4 \%$ paraformaldehyde and then blocked for 30 min using $5 \%$ donkey serum containing $0.3 \%$ Triton $\mathrm{X}-100$. The cells were then incubated with the primary antibodies against
CHGB (1:100; rabbit; PA5-52605, ThermoFisher Scientific) or SYP (1:100; rabbit; MA5-14532, ThermoFisher Scientific) for 3 and $1 \mathrm{~h}$, respectively, followed by incubation with anti-rabbit Alexa Fluor $^{\circledast} 488$ Conjugate (1:100, A11034, ThermoFisher Scientific) secondary antibody for $1 \mathrm{~h}$ at room temperature. Cells were then mounted using slow-fade DAPI (S36973, Life Technologies) and examined using Axio Observer 7.

For Adr $\beta 2$ and cytokeratin expression in tumor and adjacent normal tissues, the tissues were incubated with primary antibodies against Adr $\beta 2$ (1:100; rabbit; PA5-14117, ThermoFisher Scientific) and cytokeratin (1:100; mouse; MA1-82041; ThermoFisher Scientific) for $1 \mathrm{~h}$ at room temperature, followed by incubation with a cocktail of anti-rabbit Alexa Fluor $^{\circledR} 488$ Conjugate $(1: 100, A 11034$, ThermoFisher Scientific) and antimouse Alexa Fluor ${ }^{\circledR} 546$ ( 1 : 100, A21045, ThermoFisher Scientific) for $1 \mathrm{~h}$ at room temperature. Cells were then mounted using slow-fade DAPI (S36973, Life Technologies) and examined using Axio Observer 7.

\section{Western blotting}

Total protein from tumor and normal adjacent tissues were isolated using RIPA buffer (Thermo Scientific) containing protease and phosphatase inhibitor cocktail (Thermo Scientific). Thirty micrograms of proteins were then allowed to resolve in an SDS-polyacrylamide gel electrophoresis gel and the resolved proteins were transferred onto a polyvinylidene difluoride membrane. The membrane was then incubated with the primary antibody against Adr $\beta 2$ ( $1: 1000$; rabbit; PA5-14117, ThermoFisher Scientific) for $1 \mathrm{~h}$ followed by goat anti-rabbit horseradish peroxidase (HRP) conjugate $(1: 3000$; Biorad) and then developed using $E C L$ reagent (Biorad). The membrane was also re-probed with $\beta$-actin antibody (1: 2000; mouse; sc-47778, Santa Cruz Biotechnology) for $1 \mathrm{~h}$ followed by goat anti-mouse HRP conjugate $(1: 3000 ; 170-6516$, Biorad) and then developed using ECL reagent. The blots were visualized and images captured, using a Geldoc (Biorad).

\section{Adr $\beta 2$ inhibition studies in vitro}

For Adr $\beta 2$ inhibition studies, DU145 and LNCaP cells were pre-treated for $1 \mathrm{~h}$ with $50 \mu \mathrm{M}$ propranolol ( $\operatorname{Adr} \beta 1 / \mathrm{Adr} \beta 2$ antagonist). The cells were then treated with $50 \mu \mathrm{M}$ NE either for $6 \mathrm{~h}$ (for DU145 mRNA expression studies) or $24 \mathrm{~h}$.

\section{Orthotopic PC model}

All animal experiments were conducted after obtaining approval from the animal ethics committee at the University of Saskatchewan. Adult male athymic nude mice (Crl:NU(NCr)-Foxn1, Charles River, Canada) weighing 20- $25 \mathrm{~g}$ were used for the study and equal number of animals were randomly allocated into different groups. Tumor induction, treatment, daily monitoring of animals, and final tumor volume reading were done by the same person, with the tumor volume reading done in the presence of an additional person who had no prior knowledge of the experimental layout. The NEPC cells, LASCPC-01, were obtained from ATCC. For castration and orthotopic injection of the cells into the prostate, the mice were first anesthetized with isoflurane and injected with buprenorphine $(0.05 \mathrm{mg} / \mathrm{kg})$. A small incision was made in the abdomen, urinary bladder was exposed, and the testicles were located. Castration was performed as described previously [20], and by ligating the testicular arteries followed by removing the testicles by cutting the other end of the arteries along with the attached fat pad. Orthotopic injection was performed by injecting $2 \times$ $10^{5}$ LASCPC-01 cells in $20 \mu \mathrm{l}(1: 1 \mathrm{RPMI}$ media and Cultrex basement membrane extract (R\&D Systems)) into the exposed prostate. An additional $30 \mathrm{~s}$ was provided before removing the syringe from the prostate to minimize cell leaking. The animal's skin was sutured back and allowed to recover from anesthesia. The animals were weighed once a week. Treatment with either vehicle (saline) or propranolol $(20 \mathrm{mg} / \mathrm{kg})$ intraperitoneally was started 7 days after the tumor inoculation procedure. The treatment lasted for 21 days, and then the animals were sacrificed, and tumor sizes recorded.

\section{Statistics}

A minimum of three replicates were performed for each experiment and the exact number of replicates is given in the corresponding figure legend, excluding the samples exempted from the analysis due to technical inaccuracy. Data are presented as mean \pm SEM unless otherwise described in the figure legend. The statistical significance was calculated using standard Student's $t$-test or one-way analysis of variance followed by Dunnett's post hoc test, wherever appropriate, using GraphPad Prism ${ }^{\circledR}$ 
8.0 software. Each group in an experiment is statistically analyzed in similar manner to keep uniform variations between the experimental groups. $P$-value of $<0.05$ was considered statistically significant.

\section{REFERENCES}

1. Bray F, Ferlay J, Soerjomataram I, Siegel RL, Torre LA, Jemal A. Global cancer statistics 2018: GLOBOCAN estimates of incidence and mortality worldwide for 36 cancers in 185 countries. CA Cancer J Clin. 2018;68:394-424.

2. Patel GK, Chugh N, Tripathi M. Neuroendocrine differentiation of prostate canceran intriguing example of tumor evolution at play. Cancers. 2019;11:1405

3. Aggarwal R, Huang J, Alumkal JJ, Zhang L, Feng FY, Thomas GV, et al. Clinical and genomic characterization of treatment-emergent small-cell neuroendocrine prostate cancer: a multi-institutional prospective study. J Clin Oncol. 2018;36:2492-2503.

4. Beltran H, Prandi D, Mosquera JM, Benelli M, Puca L, Cyrta J, et al. Divergent clonal evolution of castration-resistant neuroendocrine prostate cancer. Nat Med. 2016;22:298-305.

5. Magnon C, Hall SJ, Lin J, Xue X, Gerber L, Freedland SJ, et al. Autonomic nerve development contributes to prostate cancer progression. Science. 2013;341: 1236361.

6. Zahalka AH, Arnal-Estape A, Maryanovich M, Nakahara F, Cruz CD, Finley LWS, et al. Adrenergic nerves activate an angio-metabolic switch in prostate cancer. Science. 2017;358:321-326.

7. Bautista $M$, Krishnan A. The autonomic regulation of tumor growth and the missing links. Front Oncol. 2020;10:744.

8. Barbieri A, Bimonte S, Palma G, Luciano A, Rea D, Giudice A, et al. The stress hormone norepinephrine increases migration of prostate cancer cells in vitro and in vivo. Int J Oncol. 2015;47:527-534.

9. Palm D, Lang K, Niggemann B, Drell TLT, Masur K, Zaenker KS, et al. The norepinephrine-driven metastasis development of PC-3 human prostate cancer cells in BALB/c nude mice is inhibited by beta-blockers. Int J Cancer. 2006;118:2744-2749.

10. Braadland PR, Ramberg H, Grytli HH, Urbanucci A, Nielsen HK, Guldvik IJ, et al. The $\beta(2)$-adrenergic receptor is a molecular switch for neuroendocrine transdifferentiation of prostate cancer cells. Mol Cancer Res. 2019;17:2154-2168.

11. Zahalka AH, Frenette PS. Nerves in cancer. Nat Rev Cancer. 2020;20:143-157.

12. March B, Faulkner S, Jobling P, Steigler A, Blatt A, Denham J, et al. Tumour innervation and neurosignalling in prostate cancer. Nat Rev Urol. 2020;17:119-130.

13. Dwivedi S, Krishnan A. Neural invasion: a scenic trail for the nervous tumor and hidden therapeutic opportunity. Am J Cancer Res. 2020;10:2258-2270.

14. Christie KJ, Krishnan A, Martinez JA, Purdy K, Singh B, Eaton S, et al. Enhancing adult nerve regeneration through the knockdown of retinoblastoma protein. Nat Commun. 2014;5:3670.

15. Krishnan A, Purdy K, Chandrasekhar A, Martinez J, Cheng C, Zochodne DW. A BRCA1-dependent DNA damage response in the regenerating adult peripheral nerve milieu. Mol Neurobiol. 2018;55:4051-4067.

16. Krishnan A, Zochodne DW. Is cytoplasmic PTEN a specific target for neuronal survival? Mol Neurobiol. 2015;52:1758-1764.

17. Zhang B, Wu C, Chen W, Qiu L, Li S, Wang T, et al. The stress hormone norepinephrine promotes tumor progression through $\beta 2$-adrenoreceptors in oral cancer. Arch Oral Biol. 2020;113:104712.

18. Xie T, Song $X L$, Wang $C, Y u Y Z$, Wang JQ, Chen ZS. et al. The role of androgen therapy in prostate cancer: from testosterone replacement therapy to bipolar androgen therapy. Drug Discov Today. 2021;26:1293-301.

19. Krishnan A, Hariharan R, Nair SA, Pillai MR. Fluoxetine mediates G0/G1 arrest by inducing functional inhibition of cyclin dependent kinase subunit (CKS)1. Biochem Pharm. 2008;75:1924-1934.
20. Valkenburg KC, Amend SR, Pienta KJ. Murine Prostate Micro-dissection and Surgical Castration. J. Vis. Exp. 2016. 53984. .

\section{ACKNOWLEDGEMENTS}

This work was supported by the Start-up research fund and the College of Medicine Research Award (CoMRAD) fund, from the College of Medicine, $U$ of $S$, to AK. SS is supported by the Biomedical summer research fellowship from the College of Medicine, $U$ of S. TC is supported by the Mitacs fellowship. FV and HE are supported by funds from the College of Medicine, $U$ of $S$.

\section{AUTHOR CONTRIBUTIONS}

SD performed experiments, analyzed, and interpreted the results, and composed the first draft of the manuscript. MB, SS, HE, and TC performed experiments, analyzed and interpreted the data, and made intellectual contributions. FV helped with orthotopic NEPC studies, provided intellectual contributions to the work, and helped with revising the manuscript draft. AK formulated the research question, provided sufficient resources, guided and supervised the progress of the work, and wrote the final version of the manuscript.

\section{COMPETING INTERESTS}

The authors declare no competing interest.

\section{ADDITIONAL INFORMATION}

Supplementary information The online version contains supplementary material available at https://doi.org/10.1038/s41420-021-00752-1.

Correspondence and requests for materials should be addressed to Anand Krishnan.

Reprints and permission information is available at http://www.nature.com/ reprints

Publisher's note Springer Nature remains neutral with regard to jurisdictional claims in published maps and institutional affiliations.

(C) Open Access This article is licensed under a Creative Commons C. Attribution 4.0 International License, which permits use, sharing, adaptation, distribution and reproduction in any medium or format, as long as you give appropriate credit to the original author(s) and the source, provide a link to the Creative Commons license, and indicate if changes were made. The images or other third party material in this article are included in the article's Creative Commons license, unless indicated otherwise in a credit line to the material. If material is not included in the article's Creative Commons license and your intended use is not permitted by statutory regulation or exceeds the permitted use, you will need to obtain permission directly from the copyright holder. To view a copy of this license, visit http://creativecommons. org/licenses/by/4.0/.

(c) Crown 2021 